\title{
La cristallisation et le fractionnement naturel de la matière grasse butyrique
}

\author{
par \\ C. DEROANNE \\ Chaire de Technologie Agricole et Alimentaire \\ Faculté des Sciences Agronomiques - 5800 Gembloux (Belgique)
}

\section{I. - INTRODUCTION}

L'intérêt porté au contrôle et à l'amélioration de la consistance du beurre, a suscité depuis plusieurs années l'étude de la cristallisation de la matière grasse butyrique (M.G.B.). De Man $[6,7,8,9,10,11]$ s'est particulièrement intéressé à ce problème. Il a été imité en cela par plusieurs chercheurs ; parmi ceux-ci, citons : Antila [1], Dolby [15], Lechat [20], Norris [25], Sherbon [34], Vergelesov [36]. Malgré le nombre relativement grand de travaux entrepris dans ce domaine, les connaissances théoriques sont faibles. La difficulté de synthétiser des glycérides de haute pureté et le manque de techniques analytiques appropriées, sont les principales raisons de cette déficience.

Toutefois, les principaux facteurs qui influencent la cristallisation des graisses ont été mis en évidence et depuis leur étude se poursuit. Ces facteurs sont, soit de nature chimique, composition et structure des glycérides, soit de nature physique, polymorphisme et intersolubilité. Les travaux les mieux connus, dans ce domaine, sont l'œuvre de Bailey [2], Chapman [4], Hilditch [17], Larsson [19], Lutton [21], Malkin [23], Mulder [24], Rossel [26] et Rüner [27].

L'influence du facteur chimique a été relativement peu étudiée ; les travaux se limitent à l'analyse de la composition en acides gras $[13,28,31]$ ou au dosage dez triglycérides séparés selon leur nombre de carbone par chromatographie en phase gazeuse [20]. Walstra [38] s'est préoccupé quelque peu de la présence de mono et diglycérides et de leur action comme impuretés catalytiques lors de la cristallisation de la M.G.B. Dans un prochain article nous présenterons les résultats de l'influence de la structure des triglycérides.

L'étude des facteurs physiques, en particulier le polymorphisme, a été plus approfondie. Malgré les désaccords qui persistent entre les chercheurs, à propos du polymorphisme de la M.G.B. [9], les connaissances progressent et se précisent $[34,35,40]$. Par contre, l'intersolubilité des glycérides a été très peu étudiée, bien que connue depuis longtemps. L'hypothèse émise par Mulder [24], de 
la présence de cristaux mixtes dans la graisse butyrique, n'est acceptée que depuis quelques années [12, 18, 32, 33]. L'école russe s'est particulièrement attachée à l'étude de ce phénomène [5].

Depuis plusieurs années, la cristallisation de la M.B.G. en vue de son fractionnement a été efffectuée de nombreuses fois en laboratoire [34]. L'expansion, prise actuellement par le fractionnement industriel, est liée à la nécessité de trouver de nouveaux débouchés pour la graisse butyrique, par un meilleur ajustement des propriétés de la M.G.B. pour convenir aux besoins de produits finis variés.

Les diverses méthodes de fractionnement de la graisse butyrique existant ont été répertoriées par plusieurs auteurs [13, 20, 34, 37], nous joindrons à ces bibliographies le procédé décrit par Schaap et Van Beresteyn $[28,29,30]$. Ces auteurs décrivent leur installation de fractionnement naturel de la M.G.B. sans détailler les modes de préparation des cristaux. Les méthodes industrielles mises au point sont basées sur la cristallisation fractionnée, soit de la graisse dissoute dans un solvant, soit de la graisse pure fondue. Dans ce cas, la cristallisation est suivie d'une séparation avec addition ou non d'un agent tensio-actif.

A propos de la M.G.B., il semble que le fractionnement naturel, sans solvant et tensio-actif, ait de grandes promesses d'avenir. En effet, la présence d'additif, est problématique d'un point de vue légal [37]. Le procédé Tirtiaux [14], éprouvé industriellement, est naturel ; il pourrait donc être utilisé pour " modifier " légalement (?) la M.G,B. Ce procédé est technologiquement le plus simple, le plus économique et le moins compromettant pour la saveur et l'arôme de la graisse butyrique traitée, en outre le rendement en phase solide est excellent. Cependant, ce procédé ne permet pas une diversification de la qualité des fractions aussi poussée que l'offre le fractionnement en présence de solvant. Commercialement, nous ne pensons pas que les fractions obtenues en présence de solvant soient nécessairement plus avantageuses car, par leur originalité excessive, ces fractions sont trop limitées dans leurs débouchés. Le procédé Tirtiaux n'est vraiment reproductible que pour une composition de la matière grasse relativement constante, or chacun sait que les propriétés de la M.G.B. peuvent être très différentes selon l'origine des beurres. De telle sorte que des difficultés de filtration peuvent apparaître lorsqu'il faut fractionner des M.G.B. d'origine diverse. Un autre désavantage du procédé Tirtiaux est le temps, relativement long, nécessaire au fractionnement.

Nous avons mis au point, à l'échelle pilote, un procédé de fractionnement très proche du procédé Tirtiaux, plus indépendant de la variabilité de la composition et pouvant être effectué dans les délais d'une journée de travail. Ce procédé a été mis au point grâce à une meilleure connaissance du polymorphisme et de l'inter- 
solubilité des glycérides de la M.G.B. Ce sont ces deux facteurs qui ont été plus particulièrement étudiés dans ce travail.

\section{II. - PARTIE EXPERIMENTALE}

\section{II.1. Matériel}

La mise au point de notre procédé de fractionnement, ainsi que les analyses ont été réalisées à partir de la M.G.B. obtenue après déshydratation d'un beurre d'origine hivernale. Le fonctionnement a été effectué grâce à une installation pilote de notre conception, d'une capacité de 2 , dérivée d'un cryostat et d'une unité de programmation de température «Heto ». Les fractions de la M.G.B. sont obtenues après chauffage à $60^{\circ} \mathrm{C}$ suivi d'un refroidissement jusqu'à $21^{\circ} \mathrm{C}$, température de séparation des phases solide et liquide. Le rendement, exprimé en fonction de la fraction solide, est de 29.5 p. 100.

\section{II.2. Méthodes}

\section{II.2.1 PoINT DE GOUTTE}

Le point de goutte est mesuré par la méthode Mettler, appareil Mettler FP 5 + FP 53.

La graisse fondue à $60^{\circ} \mathrm{C}$ est soumise à un refroidissement rapide à $4^{\circ} \mathrm{C}$. Le point de goutte est mesuré après un tempérage de $30 \mathrm{mn}$ à cette température. La vitesse de chauffage est de $1^{\circ} \mathrm{C} / \mathrm{mn}$.

II.2.2. Point de fusion (P.F.) ET POINT DE CRistallisation (P.C.)

Appareil Mettler FP 5 + FP 51.

L'échantillon de graisse est préparé comme décrit ci-dessus. On considère que la graisse est fondue (P.F.) dès que l'intensité de la lumière transmise atteint son maximum (fig. 1). Après cette mesure, l'échantillon est refroidi à la vitesse de $1^{\circ} \mathrm{C} / \mathrm{mn}$ pour la détermination du point de cristallisation ; celui-ci correspond à la température à laquelle l'intensité de la lumière transmise commence à diminuer (fig. 1).

\section{II.2.3. INDICE D'IODE}

Méthode de Wijs [39].

\section{II.2.4. ANALySE DES ACIDES GRAS}

Chromatographie en phase gazeuse des esters méthyliques. L'aire totale des pics des acides gras $\mathrm{C}_{4}$ à $\mathrm{C}_{18: ;}$ est sensée représenter 100 p. 100 des acides gras de la graisse. On a admis que les coefficients de réponse du détecteur FID sont identiques pour tous les acides gras. 


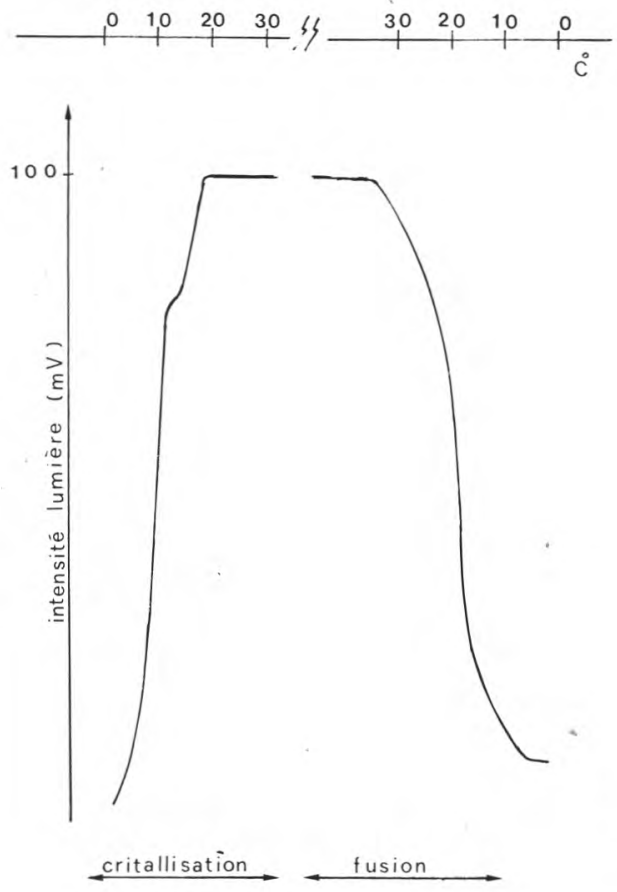

fig. 1

Courbes de fusion et de cristallisation de la matière grasse butyrique, enregistrée par l'appareil Mettler FP 5 + FP 51 .

\section{II.2.5. ANALYSE CALORIMÉTRIQUe DifFÉRENTIELLE}

Calorimètre Perkin-Elmer D.S.C. 1 B.

Mode opératoire

a) Etalonnage : les températures sont calibrées en fonction des courbes de fusion de l'indium et de la tripalmitine.

b) Prise d'essai : $10 \mathrm{mg}$ de matière grasse dans les capsules spéciales prévues à cet effet.

c) Diagramme de fusion : les capsules sont chauffées à $70^{\circ} \mathrm{C}$ puis refroidies rapidement à $-53^{\circ} \mathrm{C}$. Le diagramme de fusion est alors réalisé avec un gradient de température de $16^{\circ} \mathrm{C} / \mathrm{mn}$. Le mélange réfrigérant est composé de carboglace et d'acétone.

\section{II.2.6. DifFRaCtION DES RAYONS $\mathrm{X}$}

Diffractomètre Philips 1120, équipé d'un goniomètre PW 1050/25. Le contrôle de la température est réalisé dans une cellule porteéchantillon thermostatisée conçue par notre laboratoire [14].

Mode opératoire

Tube : $\mathrm{Cu} / \mathrm{Ni}$.

Tension générateur : $30 \mathrm{KV}$. Intensité : $24 \mathrm{~mA}$. 
Spectrogoniomètre :

- jeu de fentes : divergence $1^{\circ} \mathrm{C}$,

- réceptrice : $0,1 \mathrm{~mm}$,

- dispersion : $1^{\circ} \mathrm{C}$,

- vitesse de rotation : $1^{\circ} \mathrm{C} / 20 \mathrm{mn}$,

Compteur : HT $=1600 \mathrm{~V}$. Constante de temps : $4 \mathrm{~s}$ :

- sensibilité : $210^{-3}$.

Vitesse de déroulement du papier : $20 \mathrm{~cm} / \mathrm{mn}$.

Température échantillon : variable en fonction de l'analyse.

\section{3. Résultats}

Nous présentons les résultats de l'analyse de la graisse d'origine hivernale (A), de sa fraction liquide (L) et de sa fraction solide (S) fractionné par notre procédé.

\section{II.3.1. ANALySE Classique}

Point de goutte

Point de fusion

Point de cristallisation

Indice d'iode

Composition des acides gras principaux :

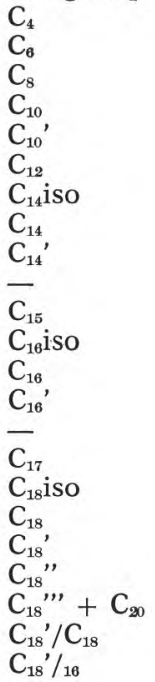

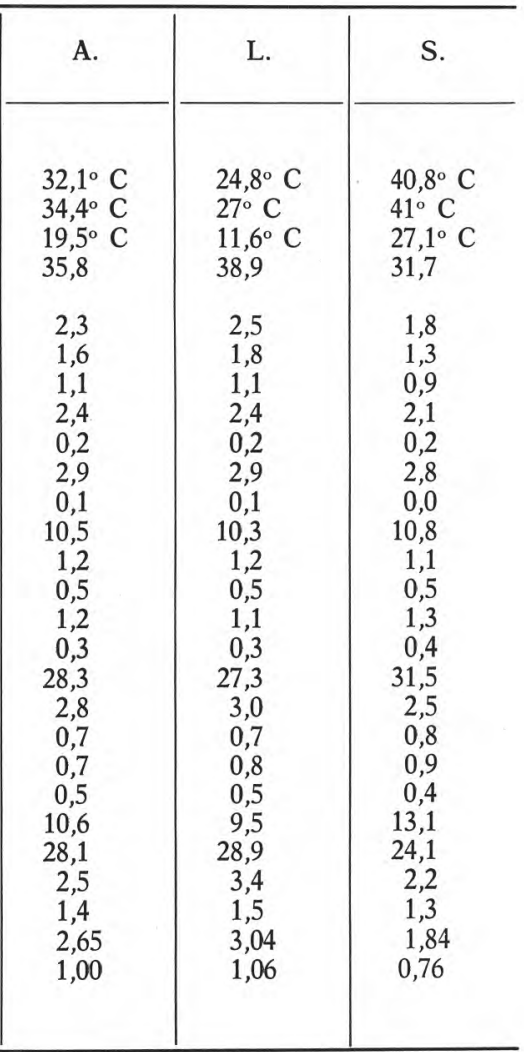




\section{II.3.2. ANAlyse CALORimétrique difFÉREnTielle (A.C.D.)}

Les diagrammes de fusion et de solidification laissent apparaître deux pics (sauf la fraction liquide) : un pic à bas point de fusion (P.B.P.F.) et un pic à haut point de fusion (P.H.P.F.) (fig. 2). Ces pics ne sont bien résolus qu'en fusion (fig. 3). Les diagrammes de fusion de la fraction solide ont été enregistrés à partir d'un échantillon non fondu $\left(S_{1}\right)$ tel qu'obtenu après filtration et également à partir d'un échantillon préalablement fondu à $70^{\circ} \mathrm{C}\left(\mathrm{S}_{2}\right)$.

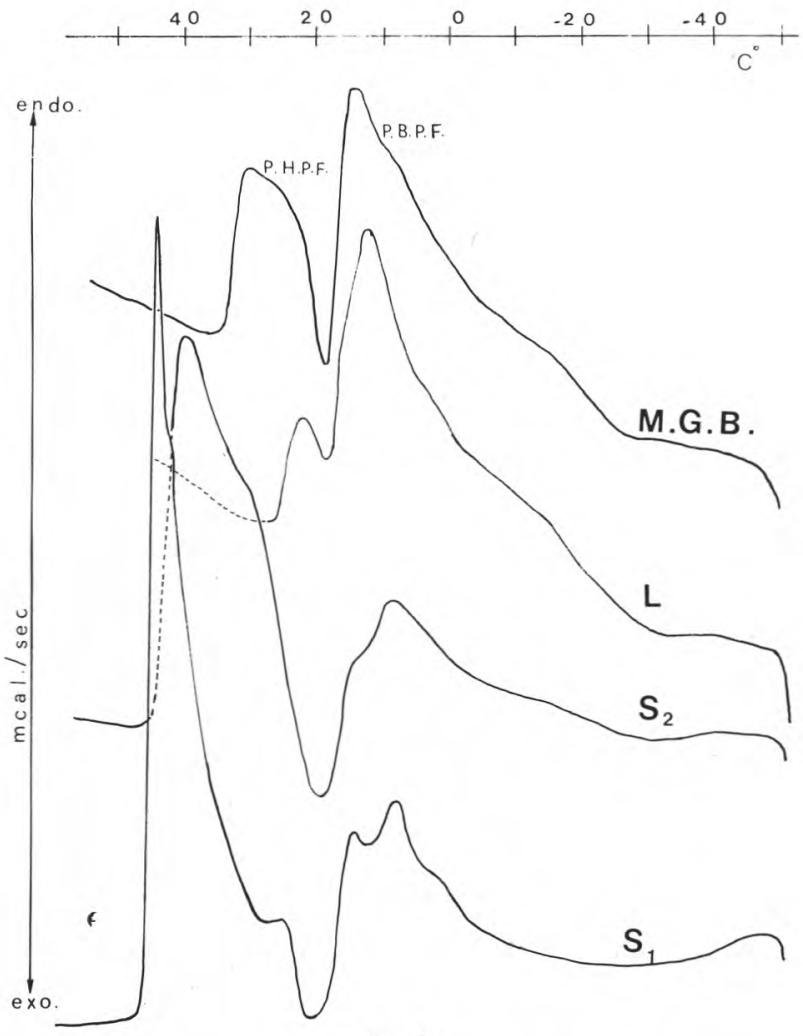

fig. 2

Diagrammes A.C.D. de fusion de M.G.B.

- Fraction liquide (L).

- Fractions solides $\left(\mathrm{S}_{1}-\mathrm{S}_{2}\right), \mathrm{S}_{1}$ : échantillon tel qu'après filtration. $\mathrm{S}_{2}$ : échantillon préalablement fondu.

Trois échantillons de M.G.B. non fractionnée ont été maintenus respectivement, lors du refroidissement, pendant $1 \mathrm{~h}$ à $25^{\circ} \mathrm{C}$, à $20^{\circ} \mathrm{C}$, à $5^{\circ} \mathrm{C}$, pour ensuite être réchauffés de $-50^{\circ} \mathrm{C}$ à $+70^{\circ} \mathrm{C}\left(\mathrm{A}_{25}-\mathrm{A}_{20}\right.$ $A_{s}$ ). Les diagrammes de fusion sont enregistrés pendant ce chauffage. 


\section{TABLEAU 1}

Zones de fusion des glycérides de la M.G.B. (A.) et de ses fractions (L et S) aires des pics de leur diagramme A.C.D.

\begin{tabular}{|c|c|c|c|}
\hline \multirow{2}{*}{ Ech. (a) } & \multicolumn{2}{|c|}{$\begin{array}{l}\text { Température de début } \\
\text { et fin de fusion }\end{array}$} & \multirow{2}{*}{$\begin{array}{l}\text { Aire } 100 \mathrm{mg} \text { de graisse } \\
\text { Aire totale } \mathrm{U}^{*} \\
\text { P.B.P.F. + P.H.P.F. }\end{array}$} \\
\hline & P.B.P.F. & P.H.P.F. & \\
\hline $\begin{array}{l}\mathrm{A} \\
\mathrm{L} \\
\mathrm{S}_{1} \\
\mathrm{~S}_{2} \\
\mathrm{~A}_{25} \\
\mathrm{~A}_{20} \\
\mathrm{~A}_{5}\end{array}$ & $\begin{array}{l}-28^{\circ} \mathrm{C}-+18^{\circ} \mathrm{C} \\
-29^{\circ} \mathrm{C}-+27^{\circ} \mathrm{C} \\
-27^{\circ} \mathrm{C}-+21^{\circ} \mathrm{C} \\
-30^{\circ} \mathrm{C}-+19^{\circ} \mathrm{C} \\
-31^{\circ} \mathrm{C}-+26^{\circ} \mathrm{C} \\
-29^{\circ} \mathrm{C}-+21^{\circ} \mathrm{C} \\
-30^{\circ} \mathrm{C}-+6^{\circ} \mathrm{C}\end{array}$ & $\begin{aligned} 18^{\circ} \mathrm{C}-34^{\circ} \mathrm{C} \\
21^{\circ} \mathrm{C}-48^{\circ} \mathrm{C} \\
19^{\circ} \mathrm{C}-44^{\circ} \mathrm{C} \\
26^{\circ} \mathrm{C}-35^{\circ} \mathrm{C} \\
21^{\circ} \mathrm{C}-34^{\circ} \mathrm{C} \\
6^{\circ} \mathrm{C}-34^{\circ} \mathrm{C}\end{aligned}$ & $\begin{array}{l}1893 \\
1770 \\
2640 \\
2774 \\
1900 \\
1967 \\
2314\end{array}$ \\
\hline
\end{tabular}

$\mathrm{U}^{*}$ : Unité d'aire arbitraire lue sur le totaliseur du planimètre.

L'aire est exprimée en fonction d'un même poids pour chaque échantillon.

(a) : (Symboles : voir texte).
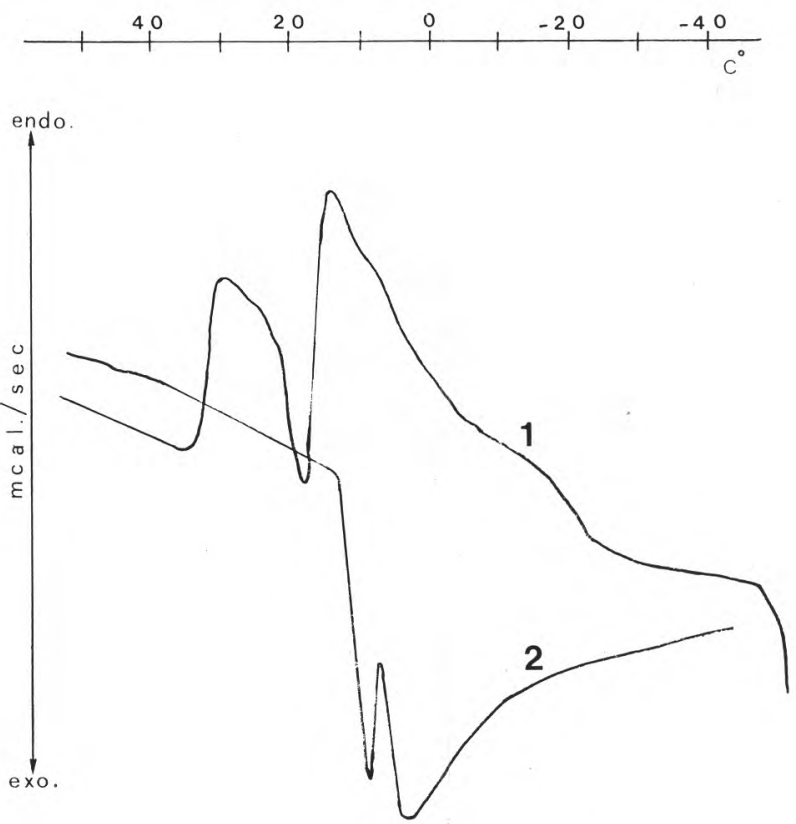

fig. 3

Diagrammes A.C.D. de fusion (1) et de solidification (2) de la M.G.B. 


\section{II.3.3. Diffraction des Rayons X (R.X.)}

Les résultats sont repris dans le paragraphe ci-dessous.

\section{II.4. Interprétation des résultats}

\section{II.4.1. ANALyse Classique}

\section{II.4.1.1. Point de goutte}

Les points de goutte sont notablement modifiés et ne correspondent plus à des valeurs normalement attendues pour des graisses butyriques pures $\left(30^{\circ} \mathrm{C}-34^{\circ} \mathrm{C}\right)$. Dans certaines limites, le fractionnement naturel permet de différencier les points de goutte de la M.G.B. et ainsi accroître, par la diversification des propriétés, les débouchés pour cette graisse.

\section{II.4.1.2. Températures de fusion et de cristallisation}

Comme les points de goutte, les températures de fusion et de cristallisation sont modifiées; ces changements sont significatifs de profondes modifications au niveau des caractéristiques physiques.

\section{II.4.1.3. Indice d'iode}

Les indices d'iode habituels des graisses butyriques sont de l'ordre de 28 à 43, les indices les moins élevés se rencontrent en hiver et les indices les plus élevés en été. Les indices d'iode des fractions de la M.G.B. peuvent être sensiblement augmentés dans la fraction liquide et diminués dans la fraction solide. Les indices restent toutefois dans les valeurs admissibles pour les graisses butyriques normales; les différences entre les valeurs ne suffisent pas à expliquer les changements des propriétés physiques.

\section{II.4.1.4. La composition des acides gras}

L'interprétation de l'analyse des acides gras de la M.G.B. a fait l'objet d'une publication antérieure [13]. Détailler ce paragraphe d'un point de vue analytique n'est donc plus nécessaire.

Nous allons préciser le rôle du polymorphisme et de l'intersolubilité dans la différenciation des compositions et des propriétés physiques des fractions de la M.G.B.

II.4.2. Analyse calorimétrique différentielle. Diffraction des RAYONS $\mathrm{X}$

Lors de l'étude de la cristallisation des glycérides de l'huile de palme [14], nous avons mis en évidence la double cristallisation. Nous avons, en effet, observé que l'huile de palme refroidie rapidement de $70^{\circ} \mathrm{C}$ à $-40^{\circ} \mathrm{C}$, se solidifie sous deux formes polymorphiques différentes, chacune de ces formes étant constituée de cristaux mixtes. Nous allons contrôler si cette observation est également valable pour la M.G.B. Pour cela, nous étudierons successivement 
le polymorphisme et l'intersolubilité ; notre étude sera basée sur les résultats de l'A.C.D, et des R.X.

\section{II.4.2.1. Le polymorphisme}

Le diagramme A.C.D. de fusion de la M.G.B. laisse apparaître deux pics. Nous allons démontrer que les glycérides de ces deux pics constituent des groupes indépendants, et que ces deux groupes de glycérides cristallisent sous deux formes polymorphiques différentes.

Lors du refroidissement de la M.G.B., préalablement fondue à $60^{\circ} \mathrm{C}$, ce sont les glycérides du pic A.C.D. à H.P.F. qui cristallisent en premier lieu, les glycérides du pic A.C.D. à B.P.F. cristalliseront après la solidification de tous les glycérides du pic à H.P.F. Cette cristallisation progressive peut être suivie par l'A.C.D. Les diagrammes A.C.D. des fractions liquides obtenues après filtration de la M.G.B. à trois températures réparties au cours du refroidissement, laissent apparaître le P.B.P.F. intact alors que le P.H.P.F. disparaît progressivement (fig. 4). La M.G.B. possède donc deux groupes de glycérides qui se caractérisent notamment par leur cristallisation dans deux zones de températures bien distinctes. Ces zones correspondent aux limites des deux pics du diagramme A.C.D. de la M.G.B.

Le polymorphisme est généralement étudié par R.X. Cette méthode, bien adaptée à l'analyse des cas simples, ne peut être utilisée que si l'échantillon est cristallisé dans une seule forme polymorphique. S'il y a plusieurs formes en présence, les R.X. ne suffisent plus et l'A.C.D. devient alors une méthode complémentaire indispensable.

Lors du refroidissement de la M.G.B. placée dans la capsule de l'appareil A.C.D. le fait de maintenir cette graisse pendant un certain temps à une température déterminée ( " tempérage ») peut nous rendre compte de la stabilité de la forme polymorphique dans laquelle la M.G.B. est cristallisée. En effet, puisque chaque transition polymorphique s'accompagne d'un accroissement de la chaleur latente de fusion et donc de l'aire du pic du diagramme A.C.D., il est possible de suivre les changements de formes. Si l'aire du pic reste constante, la forme polymorphique est stable à la température considérée. Par contre, s'il y a un accroissement de l'aire, la forme polymorphique évolue vers une forme plus stable.

Le tempérage à $25^{\circ} \mathrm{C}$ ne modifie pas l'aire des pics, la forme présente à cette température est donc stable. Par contre les tempérages à $20^{\circ} \mathrm{C}$ et à $5^{\circ} \mathrm{C}$ provoquent un accroissement de l'aire des pics, il y a donc à ces deux températures, une transition polymorphique (tab. 1).

Nous pouvons ainsi conclure que les glycérides du pic A.C.D. à H.P.F. cristallisent dans une forme stable à $25^{\circ} \mathrm{C}$ et que les 
glycérides du pic A.C.D. à B.P.F. cristallisent dans une forme instable à $20^{\circ} \mathrm{C}$ et $5^{\circ} \mathrm{C}$.

La détermination de la forme polymorphique des glycérides du P.H.P.F. (fraction solide) peut facilement être obtenue par R.X. à $20^{\circ} \mathrm{C}$ (à cette température les glycérides du P.B.P.F. sont à l'état liquide et n'interfèrent pas).

Ces cristaux du pic H.P.F. sont de la forme polymorphique $\beta^{\prime}$ (fig. 5). La nature des cristaux du pic à P.B.F. est plus difficile à déterminer. En effet, lors de l'analyse par R.X., les raies de diffraction des cristaux des glycérides des pics à H.P.F. et B.P.F. se superposent. Il faut dans ce cas se référer simultanément à l'A.C.D. et aux R.X. de la fraction liquide (P.B.P.F.). Par l'A.C.D., nous avons constaté que les glycérides du pic à B.P.F. cristallisent dans une forme instable, $\alpha$ ou $\beta^{\prime}$. La détermination de la forme polymorphique est obtenue par diffraction des rayons $\mathbf{X}$.
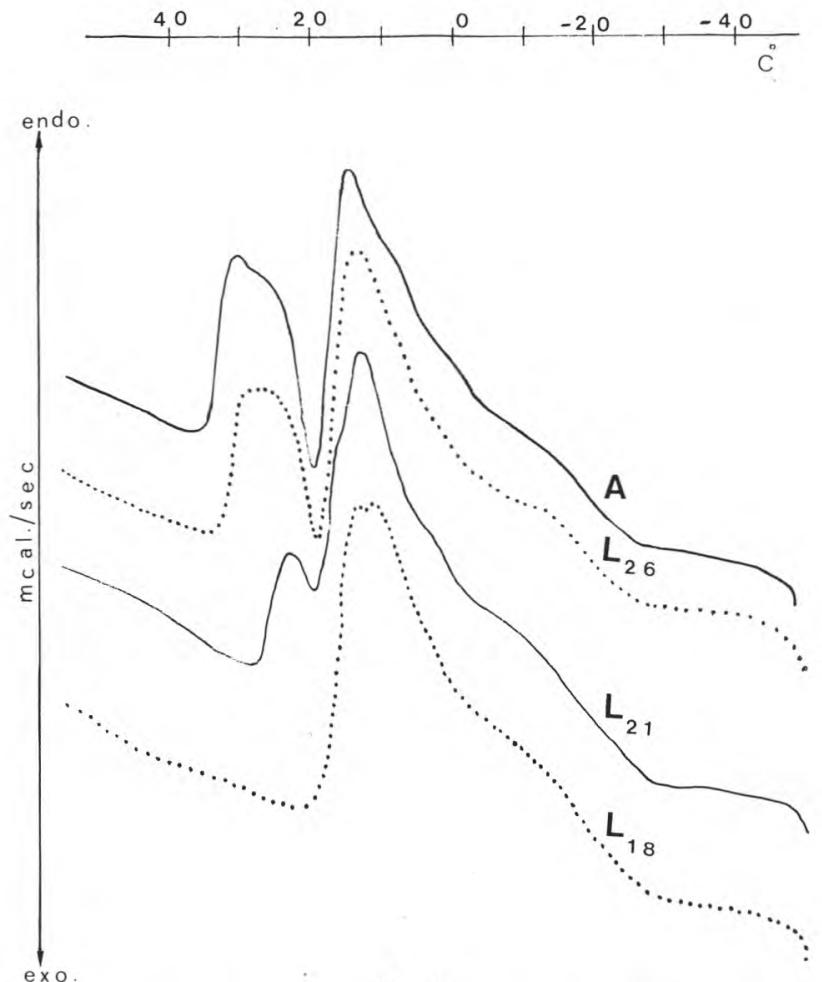

fig. 4

Diagrammes A.C.D. de la M.G.B. pure (A) et trois de ses fractions liquides filtrées à différentes températures au cours du refroidissement. 
Par notre procédé de thermostatisation de la cellule R.X., il est difficile de travailler en-dessous de $0^{\circ} \mathrm{C}$. Or à cette température une partie importante des glycérides sont à l'état liquide, et en outre, les transitions des formes polymorphiques y sont encore rapides. De ce fait, les diagrammes de diffraction R.X. sont difficiles à interpréter car, par la présence de phase liquide, les pics sont mal différenciés et les raies de diffraction de la faible portion de cristaux ayant changé de forme polymorphique se superposent aux raies de la forme initiale.

Si nous analysons par R.X. un échantillon de la fraction liquide immédiatement après son refroidissement à $0^{\circ} \mathrm{C}$, nous observons un petit pic situé à $3,83 \AA$ et un pic large et important situé à $4,13 \AA$ (fig. 5). Ce pic est caractéristique de la forme $\alpha$. Le pic à $3,83 \AA$

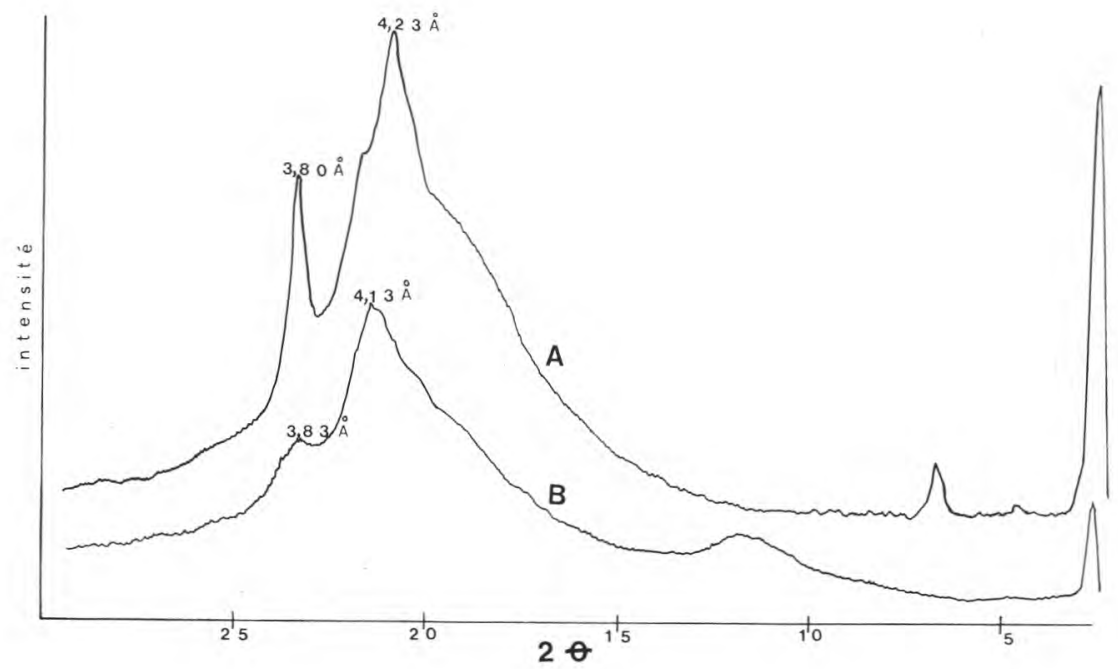

fig. 5

Courbes de diffraction des rayons $\mathrm{X}$ des cristaux des glycérides des pics A.C.D. à H.P.F. (A) et à B.P.F. (B)

pourrait être attribué en une proportion de cristaux $\beta^{\prime}$ en phase de transition $\alpha \rightarrow \beta^{\prime}$.

Les glycérides des pics A.C.D. à B.P.F. et à H.P.F. cristallisent donc respectivement en $\alpha$ et $\beta^{\prime}$. Si on tient compte des différences de chaleur latente de fusion des cristaux mixtes $\alpha$ et $\beta^{\prime}$, il est aisé de calculer, à partir du diagramme A.C.D., le pourcentage de solide. Dans le cas du fractionnement, on déterminera ainsi le rendement théorique [14]. 


\section{II.4.2.2. Intersolubilité}

Un cristal mixte résultant de l'intersolubilité de plusieurs substances se caractérise, en analyse calorimétrique différentielle, par un pic étroit semblable au pic de fusion d'un produit pur. Nous observons un diagramme de ce type pour la fraction solide non fondue après le fractionnement de la M.G.B. (fig. 2-S ${ }_{1}$ ). Le pic à H.P.F., caractéristique du cristal mixte, est accompagné d'un pic à B.P.F. dû à la présence d'huile adsorbée sur les cristaux [14]. Le pic A.C.D. résulte en fait de la fusion de plusieurs cristaux mixtes du même type. Les glycérides de la M.G.B. s'associent dans des proportions définies par les équilibres d'intersolubilité pour former un cristal mixte à H.P.F. Les glycérides à bas point de fusion, telle que la trioléine, ne sont donc pas contenus dans le cristal mixte à H.P.F. sous forme d'inclusion, Norris [25], mais comme partie intégrante du cristal. Seule une partie de ces glycérides est due à l'huile adsorbée. Les glycérides du pic A.C.D. à B.P.F. cristallisent également sous forme de cristaux mixtes.

Les deux pics du diagramme A.C.D. de la M.G.B. pure résultent donc de la fusion de deux types de cristaux mixtes solidifiés dans les formes polymorphiques $\alpha$ (P.B.P.F.) et $\beta^{\prime}$ (P.H.P.F.).

L'intersolubilité et le polymorphisme sont étroitement liés et cette relation joue un rôle important sur les propriétés physiques. La base de cette interdépendance est évidemment le fait que les glycérides d'un cristal doivent tous être cristallisés dans la même forme polymorphique. Le fractionnement naturel de la M.G.B. est un exemple pratique de cette relation entre le polymorphisme et l'intersolubilité.

\section{II.5. Le fractionnement naturel de la matière grasse butyrique}

Il est aisé de réaliser en laboratoire le fractionnement naturel d'une graisse (il suffit pour cela d'abandonner la graisse fondue à la température ambiante), par contre les difficultés apparaissent dès que l'on désire fixer des conditions reproductibles de cristallisation. La principale difficulté rencontrée lors de la réalisation industrielle du fractionnement naturel est la préparation de cristaux faciles à filtrer. La filtration constitue en effet l'opération la plus délicate. Pour obtenir par filtration la séparation des phases solide et liquide, la préparation des cristaux de morphologie adéquate est indispensable $(3,37)$. Les conditions de préparation de cristaux filtrables étant restées méconnues, le fractionnement est souvent obtenu après décantation naturelle [22]. Ce procédé est long et industriellement peu rentable, c'est pourquoi Fjaervoll [16] a mis au point un procédé de séparation par flottation en utilisant la centrifugation après addition de produits tensio-actifs. La technique de Fjaervoll est très séduisante car la nature des cristaux ne joue pratiquement 
aucun rôle. Toutefois l'ajoute de substances chimiques risque d'être problématique d'un point de vue légal.

Pour séparer les phases solide et liquide, on peut également utiliser un filtre presse, mais dans ce cas les capacités de filtration sont faibles. Actuellement, il semble que ce soit le procédé de filtration Tirtiaux, le mieux adapté au fractionnement des graisses. Tirtiaux sépare les cristaux par filtration sous vide. La filtration peut être effectuée en continu avec des capacités journalières très importantes. Toutefois le procédé Tirtiaux est subordonné à la préparation des cristaux de caractéristiques constantes bien définies. Nous avons en effet constaté, lors d'essais préliminaires, que la qualité de la séparation des fractions dépend de la forme polymorphique et de la taille des granules graisseux. Pour pouvoir être filtrés, les cristaux doivent résister au tassement et être de dimensions uniformes supérieures à $0,1 \mathrm{~mm}$, ce qui permet d'éviter le colmatage du filtre.

Les cristaux $\beta^{\prime}$, par leur structure torsadée et ramifiée [3, 14], forment des sphérolites très denses résistant bien au tassement. La taille de ces sphérolites est fonction de la vitesse de refroidissement. Lorsque les cristaux possèdent les qualités définies ci-dessus, le gâteau de filtration apparaît sec. C'est cet aspect de jaune d'œuf cuit dur, qui est caractéristique d'une bonne filtration. Le choix de la température de filtration est également très important pour la réussite d'une filtration. Successivement, nous allons détailler les conditions de préparation des cristaux $\beta^{\prime}-$ de la dimension de ces cristaux - de la température de filtration.

\section{II.5.1. PRÉPARATION DES CRISTAuX $\beta$ '}

Au paragraphe II.4.2.1., nous avons mis en évidence la cristallisation préférentielle des glycérides du pic A.C.D. à H.P.F. dans la forme polymorphique $\beta^{\prime}$. En l'absence de cristallite, la M.G.B. cristallise dans la forme $\beta^{\prime}$ si le refroidissement est rapide dans la zone des températures favorables à la transition $\beta^{\prime} \rightarrow \beta$. Une agitation trop forte dans cette même zone, favorise également la transition $\beta^{\prime} \rightarrow \beta$. Les cristallites, noyaux de cristallisation, sont présents si la température de chauffage n'est pas suffisamment élevée. En effet, ces cristallites résultent d'une fusion incomplète des cristaux [14]. Pour obtenir des cristaux $\beta^{\prime}$, il faut refroidir rapidement la graisse complètement fondue jusqu'en dessous des températures de transition, l'agitation étant ralentie avant d'atteindre ces températures.

\section{II.5.2. Dimension DES CRISTAUX}

La filtration des cristaux $\beta^{\prime}$ est rapide et le gâteau de filtration apparaît sec lorsque les sphérolites $\beta^{\prime}$ possèdent des diamètres supérieurs à $0,1 \mathrm{~mm}$. Cette condition si elle est nécessaire, ne suffit pas; il faut en outre que la taille des sphérolites soit uniforme. 
La dimension d'un sphérolite dépend de la possibilité des noyaux de cristallisation de se "nourrir " alors que l'uniformité de ces sphérolites est fonction de la régularité de la cristallisation. Il faut que, pendant le refroidissement, le nombre de noyaux de cristallisation augmente régulièrement, tout en restant faible. La croissance des sphérolites est en relation directe avec la concentration des glycérides en surfusion et également de la possibilité des glycérides de s'incorporer dans le cristal mixte. Ces deux facteurs sont cependant antagonistes. Le gradient de concentration sera d'autant plus élevé que le refroidissement est rapide, alors que les équilibres d'intersolubilité, lents à s'établir, nécessitent une diminution de la température très douce. Ce dernier facteur est donc limitatif.

L'uniformité des sphérolites nécessite également un refroidissement très lent. En effet, si le refroidissement est rapide, la forte concentration de glycérides en surfusion provoque la formation de nombreux noyaux de cristallisation et ainsi une croissance anarchique des cristaux.

Nous avons mis au point, à l'échelle pilote, les conditions d'agitation et de refroidissement favorables à la formation de cristaux faciles à filtrer (fig. 6). La qualité des fractions obtenues peut être diversifiée par la filtration à des températures différentes et par le fractionnement successif des parties liquides (tab. 2). Le fractionnement par notre procédé, peut être effectué en une journée normale de travail. Les cristaux obtenus sont faciles à filtrer, notamment par le procédé Tirtiaux. Notre procédé, ainsi que le procédé Tirtiaux

\section{TABLEAU 2}

Caractéristiques de la matière grasse butyrique (M.G.B.) et de ses fractions liquides (L) et solides (S)

\begin{tabular}{|c|c|c|c|c|}
\hline & $\begin{array}{l}\text { Point } \\
\text { de goutte }\end{array}$ & $\begin{array}{l}\text { Point } \\
\text { de fusion }\end{array}$ & $\begin{array}{c}\text { Point } \\
\text { de cristallisation }\end{array}$ & $\begin{array}{l}\text { Rdt } \\
\text { p. } 100\end{array}$ \\
\hline $\begin{array}{l}\text { M.G.B. pure } \\
\mathrm{L}_{1} \\
\mathrm{~L}_{2} \\
\mathrm{~L}_{3} \\
\mathrm{~L}_{4} \\
\mathrm{~S}_{1} \\
\mathrm{~S}_{2} \\
\mathrm{~S}_{3} \\
\mathrm{~S}_{4}\end{array}$ & $\begin{array}{l}32,1^{\circ} \mathrm{C} \\
24,8^{\circ} \mathrm{C} \\
26,7^{\circ} \mathrm{C} \\
20,1^{\circ} \mathrm{C} \\
16,6^{\circ} \mathrm{C} \\
40,8^{\circ} \mathrm{C} \\
42,6^{\circ} \mathrm{C} \\
38,6^{\circ} \mathrm{C} \\
30^{\circ} \mathrm{C}\end{array}$ & $\begin{array}{l}34,4^{\circ} \mathrm{C} \\
27^{\circ} \mathrm{C} \\
32,4^{\circ} \mathrm{C} \\
20,6^{\circ} \mathrm{C} \\
17^{\circ} \mathrm{C} \\
41^{\circ} \mathrm{C} \\
43^{\circ} \mathrm{C} \\
39^{\circ} \mathrm{C} \\
34,8^{\circ} \mathrm{C}\end{array}$ & $\begin{array}{rl}19,5^{\circ} \mathrm{C} & \mathrm{C} \\
11,6^{\circ} \mathrm{C} \\
15,5^{\circ} \mathrm{C} \\
10,2^{\circ} \mathrm{C} \\
8,3^{\circ} \mathrm{C} \\
27,1^{\circ} \mathrm{C} \\
29,7^{\circ} \mathrm{C} \\
24,7^{\circ} \mathrm{C} \\
21^{\circ} \mathrm{C}\end{array}$ & $\begin{array}{l}7 \overline{70,5} \\
83 \\
73,7 \mathrm{a} \\
64,8 \mathrm{a} \\
29,5 \\
17 \\
26,3 \mathrm{a} \\
35,2 \mathrm{a}\end{array}$ \\
\hline
\end{tabular}

a: Le rendement est calculé en fonction de la fraction liquide qui a été fractionnée. 
offrent l'avantage, bien qu'étant naturel, d'être simple et relativement rapide. La qualité des fractions est en outre stable. Au contraire de nous, le procédé de fractionnement naturel de la M.G.B., breveté par Mac Callom [22] est long et compliqué. Nous craignons que ce dernier procédé soit peu rentable industriellement car ce fractionnement nous semble être difficilement reproductible.

\section{II.5.3. TEMPÉRATURE DE FILTRATION}

Nos expériences nous ont permis de constater que le choix de la température de filtration est très important pour la réussite de la séparation des phases solide et liquide. Ainsi, avons-nous remarqué que des sphérolites, bien que possédant les caractéristiques définies ci-dessus, filtrent mal. Cet accident apparaît lorsque la filtration 'st effectuée à basse température. Dans ce cas, la filtration ralentit et peut même s'arrêter. Jusqu'à présent, on a cru que ce ralentissement de la filtration était dû à un accroissement de la viscosité. Ce brusque accroissement de la viscosité ne peut être provoqué par le seul abaissement de la température. En fait, dès que la température de refroidissement atteint la zone de cristallisation des glycérides du pic A.C.D. à B.P.F., ceux-ci, en présence d'une quantité importante d'amorces (H.P.F.) cristallisent brusquement en une multitude de petits cristaux (fig. 6). Ces petits cristaux engluent les sphérolites et colmatent progressivement le filtre. Il faut donc, lorsqu'on cristallise en une seule étape, choisir une température de filtration située au-dessus de la zone de fusion des pics à B.P.F.

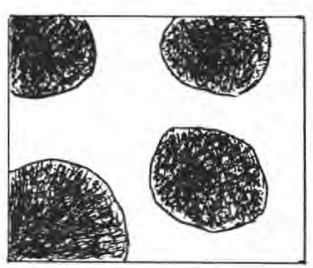

$A$

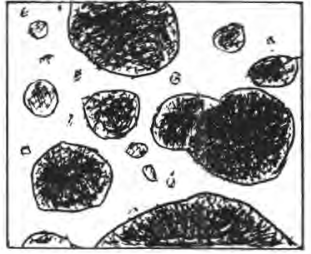

B

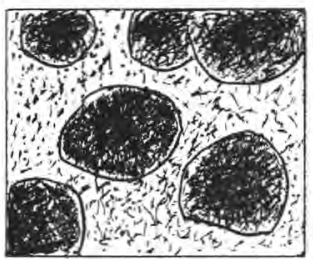

C

fig. 6

- Sphérolites réguliers (P.H.P.F.) (A) filtrent bien.

- Sphérolites irréguliers (P.H.P.F.) (B) filtrent mal.

- Sphérolites réguliers (P.H.P.F.) + petits cristaux (P.B.P.F.) (C) - le filtre se colmate.

\section{III. - CONCLUSIONS}

Les glycérides de la matière grasse butyrique déshydratée cristallisent en deux groupes de cristaux mixtes de formes polymorphiques différentes. Lors du fractionnement naturel, l'intersolubilité 
et le polymorphisme jouent un rôle très important. L'intersolubilité influence la composition des fractions alors que le polymorphisme conditionne la morphologie des cristaux et ainsi leur filtrabilité. Lors du fractionnement il faut également tenir compte de la présence de cristallites. L'analyse calorimétrique différentielle se révèle être une technique parfaitement adaptée à la mise au point du fractionnement naturel de la matière grasse butyrique. Elle permet notamment de calculer le rendement théorique d'un fractionnement.

\section{Ré s u mé}

Nous avons étudié, par analyse calorimétrique différentielle et diffraction des rayons $\mathrm{X}$, le polymorphisme et l'intersolubilité des glycérides de la matière grasse butyrique. La mise en évidence de la double cristallisation dans les formes polymorphiques $\alpha$ et $\beta^{\prime}$ de deux types de cristaux mixtes a été utilisée pour préciser les conditions du fractionnement naturel de la matière grasse butyrique déshydratée.

\section{S u m m a r y}

The polymorphism and the intersolubility of milk fat was studied by differential scanning calorimetry and $\mathrm{X}$ ray diffraction. The double crystallization in the polymorphic forms $\alpha$ and $\beta^{\prime}$ of two mixed crystals are used to stade the procedure of the natural fractionation of butter oil.

\section{Remerciements}

Nos remerciements s'adressent à $M$. P. Jamotte, Directeur de la Station Laitière de Gembloux, ainsi qu'à $M$. le professeur $V$. Tonnard, Chaire de Sciences du Sol de la Faculté Agronomique de Gembloux, qui nous ont permis de travailler dans leurs laboratoires et de disposer de l'appareil d'analyse calorimétrique différentielle et de la diffraction des rayons $X$.

Reçu pour publication en août 1975.

\section{Bibliographie}

[1] Antila (V.) (1966). - Meijeriteit. Aikak, 27, 1.

[2] Bailey (A. E.) (1950). - Melting and Solidification of fats.Interscience Publ.

[3] Beyerlein (V.) und Voss (E.) (1973). - Kieler Milch. Forsch., 25, 49.

[4] Chapman (D.) (1962). - Chem. Rev., 433.

[5] XVIII Congrès International de Laiterie, Sydney, 1970. 
[6] De Man (J. M.) (1958). - J. Dairy Sci., 41, 360.

[7] De Man (J. M.) (1959). - J. Dairy Sci., 42, 56.

[8] De Man (J. M.) (1961). - J. Dairy Res., 28, 81.

[9] De Man (J. M.) (1961). - J. Dairy Res., 28, 117.

[10] De MAN (J. M.) (1962), - XVI e Congrès Int. de Lait., Copenhague.

[11] De Man (J. M.) (1963). - Dairy Sci. Abstracts, 25, 219.

[12] Deroanne (C.) et Severin (M.) (1973). - Lebensm. Wiss. u. Technol., 6, 30.

[13] Deroanne (C.) et Guyot (A.) (1974). - Bull. Rech. Agron. Gembloux, 9, 3.

[14] Deroanne (C.) (1975). - Thèse de doctorat, Gembloux.

[15] Dolby (R. M.) (1970). - XVIII Congrès Int. de Lait., Sydney.

[16] Fuaervoll (A.) (1970). - Dairy Industries, 3, 8, 502.

[17] Hilditch (T. P.) (1964). - The Chemical Constitution of Natural Fast, 4 ed., Chapman et Hall, London.

[18] Krautwurst (J.) (1970). - Kieler Milch. Forsch., 22, 255.

[19] Larsson (K.) (1972). - Fette Seifen Anstrichmittel, 74, 136.

[20] Lechat (G.) et al. (1975). - Le Lait, 545-546, 295.

[21] Lutron (E. S.) (1972). - J. Am. Oil Chem. Soc., 49, 1.

[22] Mac Collom (M. S.) (1970). - U.S. Patent, 3, 5, 19, 435, 7 juillet.

[23] MaLkin (T.) (1954). - Progress in the Chemistry of fats and other lipids, Academic Press.

[24] Mulder (H.) (1953). - Neth. Milk Dairy J., 7, 149.

[25] Norris (R.) et al. (1971). - J. Dairy Res., 38, 179.

[26] Rossel (J. B.) (1965). - Advances in Lipid Research, Ed. R. Paoletti and D. Kritchevsky, V. 5.

[27] RüNER (U.) (1971). - Lebensm. Wiss. u. Technol., 4, 76-81-113-175.

[28] SchaAP (J. E.) et VAN BERESTEYN (E.C.H.) (1970). - Voedingsmiddelentechnologie, $1, \mathrm{n}^{\circ} 46$.

[29] SchaAp (J. E.) et VAN Beresteyn (E.C.H.) (1971). - Voedingsmiddelentechnologie, $2, \mathrm{n}^{\circ} 47$.

[30] SchaAp (J. E.) et RuITtEN (G.A.M.) (1974). - Voedingsmiddelentechnologie, $7, n^{\circ} 39,8$.

[31] Schulz (M. E.) (1967). - Kieler Milch. Forsch., 16, 279.

[32] Sherbon (J. W.) and Dolby (R. M.) (1972). - J. Dairy Res., 39, 319.

[33] Sherbon (J. W.) et al. (1972). - J. Dairy Res., 39, 325.

[34] Sherbon (J. W.) (1974). - J. Am. Oil Chem. Soc., 51, 22.

[35] Van Beresteyn (E. C. H.) (1972), - Neth. Milk Dairy J., 26, 117.

[36] Vergelesov (V. M.) (1970). - XVIII ${ }^{e}$ Congrès Int. de Lait., Sydney.

[37] Voss (E.) et al. (1971). - Milchwissenschaft, 26, 605.

[38] Walstra (P.) und Van Beresteyn (E. C. H.) (1975). - Neth. Milk Dairy J., 29,35 .

[39] WolfF (J. P.) (1968), - Manuel d'Analyse des Corps Gras, Azoulay, Paris, p. 115 .

[40] Woodrow (T. L.) and De Man (J. M.) (1968), - J. Dairy Sci., 51, 996. 